\title{
Interacciones Alumnos-Profesor en Contextos de Vulnerabilidad Respecto de la Ocurrencia de Episodios de Violencia Simbólica en Clases de Matemáticas
}

\author{
Abelardo M. Castro ${ }^{(1)}$, Eduardo E. Mardones ${ }^{(2)}$, Andrés I. Ortiz ${ }^{(3)}$, Fabián E. Quiroga ${ }^{(3)}$ \\ (1)Depto. Ciencias de la Educación, Facultad de Educación, Universidad de Concepción, Barrio \\ Universitario s/n, Casilla 160-C, Concepción-Chile (email: acastro@udec.cl). \\ (2) Depto. Metodología de la Investigación e Inf. Educacional, Facultad de Educación, Universidad de \\ Concepción, Barrio Universitario s/n, Casilla 160-C, Concepción-Chile.(email: emardone@udec.cl) \\ (3) Depto. Curriculum e Instrucción, Facultad de Educación, Universidad de Concepción, Barrio \\ Universitario s/n, Casilla 160-C, Concepción-Chile.(email: aortiz@udec.cl, fquiroga@udec.cl)
}

Recibido Jul. 31, 2012; Aceptado Sep. 14, 2012; Versión final recibida Nov. 05, 2012

\begin{abstract}
Resumen
Se presenta un estudio que propone identificar y caracterizar cómo se manifiesta la contradicción entre el habitus del estudiante respecto del razonamiento matemático y la actividad didáctica realizada por el profesor en la implementación del curriculum en clases de matemática. La investigación es de carácter cualitativo y es parte del proyecto "Dialéctica de los aprendizajes y ruptura del círculo de bajos aprendizajes en sectores de alta vulnerabilidad". El estudio está basado en análisis de clases de matemáticas de quinto básico y permitió detectar manifestaciones de violencia simbólica por parte del profesor cuando invalida el razonamiento matemático de los estudiantes. Este estudio concluye que en contextos de vulnerabilidad el proceso de construcción del conocimiento matemático se desvincula del capital cultural de los estudiantes. Así mismo la existencia de manifestaciones de violencia simbólica dificulta el razonamiento matemático de los estudiantes, pues oculta su capital cultural y legitima el razonamiento impuesto por el profesor.
\end{abstract}

Palabras clave: violencia simbólica, razonamiento matemático, habitus, vulnerabilidad, capital cultural

\section{Student-Teacher Interactions in Vulnerability Contexts and the Occurrence of Episodes of Symbolic Violence in a Course Of Mathematics}

\begin{abstract}
This paper presents a study that proposes to identify and characterize the contradiction between the student's habitus regarding mathematical reasoning and the teacher's didactic action in a course of mathematics of an elementary school. This is a qualitative research and is part of the major project "Dialectics of learning and breaking the low achievement circle in highly vulnerable areas". The study is based on the analysis of the fifth grade of elementary mathematics and allowed identifying symbolic violence of the teacher when his way of teaching invalidates the student's mathematical reasoning. The study concludes that, in vulnerability contexts, the process of construction of mathematical knowledge is detached from the student's cultural capital. Similarly, the manifestation of symbolic violence makes it difficult the student's mathematical reasoning since it hides their cultural capital and legitimizes the reasoning imposed by the teacher.
\end{abstract}

Keywords: symbolic violence, mathematical reasoning, habitus, vulnerability, cultural capital 


\section{INTRODUCCIÓN}

Actualmente en Chile, los resultados de mediciones estandarizadas como el Sistema de Medición de la Calidad de la Enseñanza (SIMCE) ponen de manifiesto una realidad preocupante en el sistema educativo respecto al subsector matemática, la cual señala que los alumnos y alumnas de contextos socioeconómicos vulnerables tienen desempeños en matemática significativamente más deficientes que los estudiantes de sectores no vulnerables. Dicha relación se explica comúnmente asumiendo que en sectores de pobreza económica se manifiestan carencias de carácter cultural en estos estudiantes, las cuales les impiden desarrollar habilidades relacionadas con el aprendizaje de la matemática, lo cual finalmente se traduce en logros de aprendizaje deficitarios.

El análisis de las teorías sociológicas relacionadas con la caracterización del capital cultural del estudiante de escuelas en contextos de vulnerabilidad, permiten entender desde una perspectiva teórica distinta a las llamadas "carencias culturales" que supuestamente estarían presentes en estos estudiantes. Lo anterior permite entender que los estudiantes en contexto de vulnerabilidad poseen un capital cultural distinto al capital cultural que la escuela valora y que se reconoce, por tanto, lo que se manifiesta en las salas de clases en dichos contextos es una encuentro de culturas distintas que se relacionan en el ejercicio de la implementación del curriculum escolar de matemática. Respecto de este encuentro de culturas y su manifestación en la interacción profesor alumno, se destaca la no existencia de oportunidades que permitan considerar las producciones de los estudiantes en las clases de matemática.

Al analizar estos episodios con respecto del concepto de violencia simbólica desarrollado por Bourdieu, (Bourdieu, 1977) se hace posible concluir que en las clases de matemática observadas se imponen formas de razonamiento arbitrarias que no validan ni consideran las formas de razonamiento matemático que manifiestan los estudiantes en sus producciones, por tanto, desde el punto de vista de Bourdieu se estaría en presencia de situaciones proclives a generar episodios de violencia simbólica.

Por otra parte, desde el punto de vista de la Teoría Antropológica de lo Didáctico desarrollada por Yves Chevallard (Chevallard,1999) permite entender que, si las formas de razonamiento son efectivamente impuestas por el profesor en la clase de matemática generando manifestaciones de violencia simbólica, éstas responden a vestigios sobre su pensamiento, conocimiento y creencias respecto del contenido matemático y su forma de enseñar. Lo anterior desvincula al estudiante del proceso de construcción del conocimiento matemático, dejando a su capital cultural relegado en segundo plano respecto a su utilidad en el proceso de aprendizaje producto de su incompatibilidad con el conocimiento pedagógico de la matemática que posee el docente, el cual se ve influenciado por textos de estudio, estándares nacionales de desempeño y el currículo. Todo lo anterior podría traducirse en la inexistencia de la transposición didáctica necesaria para generar aprendizajes en matemática.

En la presente investigación se espera dar respuesta a las siguientes interrogantes:

a. ¿Qué caracteriza a las manifestaciones de violencia simbólica en las clases de matemática de 5to año básico en establecimientos de vulnerabilidad social?

b. ¿Existe una contradicción entre el habitus del alumno respecto de su razonamiento matemático y la forma como el profesor enseña la matemática del currículum escolar?

c. ¿Cómo se manifiesta la contradicción entre el habitus del alumno respecto a su razonamiento matemático y la actividad didáctica realizada por el profesor en la implementación del currículum escolar?

En relación a las preguntas propuestas anteriormente se plantean los siguientes objetivos para guiar el desarrollo de investigación:

a. Identificar los episodios de violencia simbólica que se generan en el aula en el subsector de educación matemática en quinto año básico.

b. Caracterizar de qué forma los episodios de violencia simbólica se manifiestan en la interacción profesor alumno.

c. Describir de qué forma los episodios de violencia simbólica se relacionan con la implementación del currículum escolar en matemática.

\section{ANTECEDENTES}

En el contexto de los resultados que ofrece el Sistema de Medición de la Calidad de la Enseñanza (SIMCE) se pone de manifiesto que los alumnos y alumnas de escuelas en contextos socioeconómicos vulnerables tienen desempeños en matemática significativamente más deficientes que los estudiantes de sectores no vulnerables (MINEDUC, 2010). Específicamente en el sector matemática en 4to año de formación general 
básica nivel escolar para niños y niñas de entre 9 y 10 años de edad, siendo los resultados obtenidos por los estudiantes del grupo socioeconómico bajo en la medición SIMCE entre los años 2002 y 2010 alcanzan los 220 puntos con una desviación típica de 52 puntos.

Estos establecimientos considerados vulnerables, que se caracterizan por tener bajas de matrículas en años consecutivos, trabajar con estudiantes provenientes de familias de escasos recursos económico, que viven en sectores de deprivación social, tener gran tasa de estudiantes con problemas de indisciplina y baja autoestima, apoderados con bajo nivel de escolaridad y participación en la formación de los estudiantes, baja tasa comparativa de logros en resultados SIMCE.

Al comparar dicho datos con el obtenido por los estudiantes del grupo socioeconómico alto en igual periodo alcanzaron los 300 puntos con una desviación típica de aproximadamente 43 puntos.

Teniendo presente estos antecedente y comprendiéndolos desde el punto de vista del nivel de logro de los aprendizajes que reflejan los puntajes antes presentados, es posible determinar que los estudiantes del grupo socioeconómico alto han logrado los desempeños esperados para su nivel escolar, específicamente implica que estos alumnos y alumnas que alcanzan dichos logros demuestran:

a. Conocimiento básico del sistema de numeración decimal, al comprender el valor posicional de los dígitos que forman un número natural.

b. Utilización de fracciones para cuantificar partes de una unidad.

c. Organización de información en formatos simples y elaboran nueva información a partir de datos dados.

d. La capacidad de caracterizar y relacionar formas geométricas a partir de sus elementos y reconocen movimientos en el plano.

e. Realizar cálculos con números naturales, utilizando los algoritmos convencionales.

f. Resolver problemas sencillos que requieren idear un procedimiento de resolución.(MINEDUC, 2008)

Por otra parte, los estudiantes pertenecientes al grupo socioeconómico bajo poseen un nivel de logro inicial de los desempeños esperados en el subsector lo que demuestra un conocimiento limitado de los temas relevantes del subsector de estudio y no muestran habilidades matemáticas consolidadas respecto a los contenidos del nivel. Lo anterior implica de forma específica, que los alumnos y alumnas de este grupo socioeconómico bajo "recién están iniciando la comprensión de los números naturales, la realización de los cálculos simples, el estudio de las formas geométricas y el manejo de aspectos básicos de la resolución de problemas" (MINEDUC, 2008)

Una forma de aproximación a los posibles motivos por los cuales se podría generar esta diferencia en los niveles de aprendizaje de los estudiantes, se centra en el análisis y caracterización del capital cultural (Bourdieu, 1977) de los estudiantes de escuelas en contextos de vulnerabilidad y su relación con la cultura que la escuela valida y reconoce en las distintas instancias de interacción social que se ejercen a partir de la acción educativa. Dicho análisis permite entender ambos contextos, el asociado al capital cultural de los estudiantes y la cultura escolar oficial, con centro en las características de cada una de ellos, más que en la determinación de carencias culturales que supuestamente estarían presentes en los estudiantes.

Desde esta perspectiva, es posible determinar que "los estudiantes en contexto de vulnerabilidad poseen un capital cultural distinto al capital cultural que la escuela valora y reconoce" (Castro 2009) por tanto en la ejecución de cualquier acción pedagógica se genera un encuentro de culturas distintas en el aula que se relacionan en la implementación de un curriculum escolar, en este caso específico, en el sector matemática.

Otro elemento que permite entender el encuentro cultural en las clases de matemática es a partir del análisis del habitus (Bourdieu 1977) de los diferentes actores y cómo este se manifiesta en las clases de matemática. En el caso de los estudiantes, el estudio de su habitus permite captar de una forma más amplia los distintos modos de estructurar significados en las clases de matemática y al mismo tiempo determinar cómo estos significados son utilizados por los estudiantes a la hora de necesitar responder a una tarea matemática tratada en clases o a una situación cotidiana en la que se requiera de la utilización del conocimiento matemático específico para su interpretación.

Desde la perspectiva de la manifestación del habitus por parte del profesor, su estudio entrega indicios de la apropiación práctica de los esquemas que sirven para producir las prácticas adecuadas a una situación de gestión de clases; en este sentido, la forma en que el docente gestiona las interacciones con sus estudiantes al interior del aula permitirá tener información respecto a los esquemas que estructuran su idea de clase de matemática y también su visión respecto a lo que implica para el docente la matemática y su enseñanza. 
Una aproximación a la descripción del encuentro cultural que se produce en las aulas de colegios en contextos de pobreza económica queda de manifiesto en los aportes de (Willis, 1988) cuando se describe la resistencia activa hacia la escuela de estudiantes de sectores obreros de Inglaterra. Willis compara la cultura escolar y la cultura de los obreros, determinando las características de ambas en términos de la predominancia y la valoración de la forma en cada uno de estos sectores concibe la ejecución de una tarea específica. En el caso de la cultura escolar, la ejecución de una tarea específica se manifiesta como teórica con énfasis en su análisis y búsqueda de patrones generalizables que respetan axiomas previamente definidos, sin embargo en la segunda, la cultura de los obreros, se presenta principalmente con foco en la práctica, asignando valor exclusivo al logro de la tarea y su funcionalidad. Bajo estas condiciones el encuentro cultural estará influenciado por continuas tensiones respecto a lo que se espera se aprenda en clases (lo por aprender, reflejo de la cultura escolar) y lo que el estudiante posee como capital cultural (lo aprendido, reflejo de la herencia cultural del estudiante).

Una manifestación de estas tensiones generadas en el desarrollo de clases de matemática podrían reflejarse en la forma en que el docente utiliza las producciones que los estudiantes elaboran al ejecutar una tarea matemática específica (Chevallard, 1999). Según Chevallard la ejecución por parte de los estudiantes de una tarea matemática a partir de la utilización de una técnica específica, siempre estará acompañada de vestigios de un discurso racional asociado a la justificación de la utilización de dicha técnica. El origen de esta explicación racional podrá ser diversa, la propia escuela, pero también en entornos no necesariamente escolarizados. Sin embargo, independiente del origen de dicha explicación, la ejecución de una tarea matemática por parte del estudiante manifiesta la elaboración de un producto que contendrá elementos asociados a su habitus. En consecuencia, es probable que el docente espere que sus estudiantes elaboren una producción con centro en el análisis teórico de los principios que justifican la realización de la tarea, sin embargo es esperable que los estudiantes en contextos vulnerables, no respondan a este patrón de acción, principalmente porque su habitus es netamente práctico con centro en la concreción de la tarea más que en la justificación del porqué la técnica "funciona" para la elaboración de una respuesta correcta para dicha tarea. En este sentido, las tensiones podrían emerger ya que por una parte el profesor no recibe el producto esperado y, por su parte, el estudiante no percibe que su actuación en el desarrollo de la tarea matemática sea valorada.

Los discursos racionales que se espera que los estudiantes manifiesten en el proceso de justificación de la utilización de una técnica determinada en matemática, podrán ser analizados también desde la perspectiva del modelo de tratamiento del discurso planteado por Bourdieu (1985). Este análisis se presenta como un resultado de la relación entre el habitus lingüístico y el mercado lingüístico presentes en el proceso de interacción entre profesor y estudiantes desde el punto de vista del modelo económico de los intercambios lingüísticos (Bourdieu ,1985). Desde esta perspectiva el valor que pueda tomar un determinado discurso en una clase de matemática estará determinado por las leyes que se definan en este mercado lingüístico específico en el cual interactúan leyes de aceptabilidad de dichos discursos y las prácticas lingüísticas, los cuales quedarán definidas implícita o explícitamente por el docente.

Al aplicarse estas leyes sobre los discursos planteados por los estudiantes en el contexto de una argumentación matemática, éstos quedarán expuestos a la valoración que se haga de ellos respecto a las leyes ya establecidas, emergiendo de dicha valoración relaciones de fuerza y dominación. Dicha valoración, en el contexto de este estudio, podrá estar dada por el docente que gestiona las clases de matemática, el cual puede hacer valer su posición consolidada al interior del aula respecto de sus estudiantes en forma de estrategias expresivas como hipercorrección, hipocorrección controlada la informalidad o la "campechanía que muestran los que están en posiciones muy seguras de su dominio social para hacer observar que tienen poder para eludir la norma lingüística o simbólica en su provecho" (Alonso, 2004). Estas formas de comunicación por parte del docente manifestarían las característica de su habitus lingüístico el cual, según lo descrito anteriormente podrá relacionarse de forma contradictoria o complementaria con el capital cultural de los estudiantes.

Desde la perspectiva del estudiantes, su discurso emitido tendrá un valor que dependerá de la "habilidad que tenga cada sujeto de convencer a sus virtuales receptores de la autoridad, legitimidad y ajuste a las fuentes de poder de su discurso específico" (Alonso, 2004). Esto implica que el valor del discurso que pueda emitir un estudiante en la sala de clases dependerá de las leyes que caracterizan el mercado Lingüístico determinado por la clase de matemática y la relación de fuerza que se establezcan efectivamente entre las competencias lingüísticas de producción y apropiación de los capitales simbólicos valorados y validados en la clase de matemática.

Si consideramos que el aprendizaje se puede concebir como "un hecho interactivo intra e inter sujetos, en el que el lenguaje es herramienta de apropiación y desarrollo de conocimiento" (Villalta y Martinic, 2009) y por tanto la forma en que se construya este conocimiento estará relacionado con la forma en que se establezca 
la interacción entre profesor y estudiante respecto a la tarea matemática planteada. Desde esta perspectiva, el análisis de estos discursos que se estructuran en forma y contenido de manera distinta a otros contextos de interacción social, permitirán contribuir a la caracterización de la forma en que se establecen las interacciones al interior del aula como medio de generación y reconocimientos del razonamiento matemático de los estudiantes.

Según los argumentos antes expuestos, es posible entender qué el contexto en que se ejecuta el proceso de enseñanza se relaciona con la interacción que se genera al interior del aula respecto a la manifestación de culturas distintas. En este sentido la visión del aprendizaje significativo (Ausubel, 2002), se complementa con la presentada por Castro, (2009), la cual señala que existe una relación dialéctica entre lo aprendido y lo por aprender, que puede ser de tipo complementaria (como en el caso del aprendizaje significativo), neutral, contradictoria o contradictoria antagónica; en este sentido, la posibilidad de generar una relación complementaria entre lo aprendido y lo por aprender en el estudiantes en clases de matemática estará fuertemente relacionada con la consideración y validación del capital cultural del estudiante en el proceso de transposición didáctica (Chevallard, 1999).

Por el contrario, si en el proceso de enseñanza se caracteriza por la ausencia de la consideración del capital cultural del estudiante en el proceso de gestión de clases de matemática, es probable que la elaboración de conceptos y procedimientos en la clase de matemática, estén fuertemente relacionados con la imposición de significados de forma arbitraria con alto valor teórico y práctico para la matemática y el docente, pero sin mayor significación para el estudiantes. De esta forma, la acción pedagógica impone significados y esconde en esa legitimidad el carácter arbitrario de éstos sin considerar el capital cultural de los estudiantes, lo cual impide el desarrollo de relaciones complementarias entre lo aprendido y lo por aprender. En este sentido, la no consideración de posibles argumentaciones, significados y procedimientos alternativos, y la posterior imposición de significados de forma arbitraria, manifiesta la presencia de episodios de violencia simbólica (Bourdieu, 1977) en el contexto de clases en matemática.

\section{METODOLOGÍA}

El presente estudio está adscrito a un diseño de investigación de carácter cualitativo bajo un propósito exploratorio de carácter descriptivo basado en la observación directa en la sala de clase. Para la concreción de esta investigación se consideraron los siguientes parámetros:

Como sujetos de estudio se consideraron los profesores de establecimientos que participan en el Proyecto Anillos Conicyt SOC-15, los cuales alcanzaron a la cantidad de siete escuelas pertenecientes a la Provincia de Concepción Chile, que se consideraron adscritos bajo el concepto de establecimientos de carácter vulnerables. Para efectos de esta investigación la muestra ha sido de carácter intencionada, siendo un muestreo comprensible que se realizó a partir de criterios y escenarios en donde tenían lugar los acontecimientos, es decir en contextos vulnerables dónde se desarrollaban habilidades de razonamiento matemático. Ello llevo a considerar tres establecimientos en dónde se realizaron las acciones que se describen más adelante.

Los participantes considerados en este estudio fueros tres profesores que realizan clases de matemática en el nivel Quinto año de Educación General Básica en tres establecimientos independientes y adscritos a la investigación cuyos niveles socioeconómicos y cultural los definen con el carácter de establecimientos de alta vulnerabilidad. Los profesores seleccionados poseen diferentes edades lo cual se relaciona con los años de experiencia en el ejercicio de la pedagogía.

Con la intención de profundizar en la forma en que se manifiesta dicho encuentro de culturas en la clase de matemática, se observaron clases de estudiantes pertenecientes a la muestra. Dicha observación se enfocó especialmente en el análisis de la interacción profesor - estudiantes en el desarrollo de tareas matemática y cómo en esta interacción emergen distintas manifestaciones del capital cultural que caracterizan el proceso de gestión de clases en matemática en ese nivel educativo.

A partir de las grabaciones y uso posterior de tres videos, el análisis de estas clases se realizó con el fin de identificar y caracterizar los episodios de violencia simbólica centrados en la no consideración del razonamiento matemático de los estudiantes. Ello permitió detectar la existencia de diferentes formas de interacción que asume el profesor con sus estudiantes en el desarrollo de las clases de matemática respecto de las tareas que el docente solicita a sus estudiantes y la posterior utilización de dicha información en el desarrollo de la clase. Operativamente la organización de la metodología de trabajo se estructuró a partir de fases que se explicitan a continuación.

Primera fase: Se consideraron los establecimientos participantes así como los tres docentes que realizaran las clases de Educación Matemática en $5^{\circ}$ año de Educación General Básica en dichos establecimientos, 
quienes mostraron interés en la investigación. En consecuencia se consideraron a los alumnos participantes por curso de dichos docentes.

Segundo fase: Se estableció una entrevista con los Directores de los establecimientos participantes, con la finalidad de establecer una primera aproximación con las escuelas así como también con los docentes participantes, la cual culminó con un protocolo de acuerdo en dónde se acepte los procesos de observación y grabaciones a nivel de aula de las actividades de matemática a realizar por los docentes.

Tercera fase: Se entrevistó a los docentes participantes con la finalidad de dar a conocer el procedimiento a ser aplicado durante el proceso de filmación de la clase y la fecha en que se desarrollará dicha filmación.

Cuarta fase: En esta fase se inició el proceso de filmación de las clases realizadas por los profesores participantes en sus respectivos establecimientos.

Quinta fase: Se destinó a la observación de las filmaciones por parte del Equipo investigador anillo subsector Matemática, de igual forma, se realizó la elaboración de un instrumento matriz que permitió llevar un registro escrito de los episodios como la acción docente, las cuales permitirán apreciar situaciones asociadas a episodios de clases que se relacionan con formas de violencia simbólica en las filmaciones realizadas a cada docente participante.

Sexta fase: A partir de las filmaciones efectuadas se procedió a realizar un proceso de completación de la matriz de análisis, la cual permitió realizar el seccionamiento de las filmaciones, posibilitando así la identificación de las acciones que mejor den muestra de la violencia simbólica identificada. Dicha matriz permitió organizar, sistematizar y analizar las acciones docentes gestionadas en la sala de clases y filmadas; de esa forma se obtuvo evidencias de forma contextualizada respecto de los episodios individuales de violencia simbólica generados durante el proceso de aprendizaje.

Séptima fase: En esta fase se procedió a realizar la recolección de episodios que dan evidencia audiovisual de la violencia simbólica observada, producto de las acciones docentes a nivel de aula.

Octava fase: Elaboración de informe final.

\section{RESULTADOS}

Para la descripción de los principales resultados de la investigación, se efectuará de forma secuencial respecto de los episodios observados en las clases de matemática en los diferentes establecimientos de la muestra, los cuales se identificarán con las letras A, B y C en relación a las categorías asociadas con el no reconocimiento de las producciones matemáticas realizadas por los estudiantes que emergen del análisis de los datos, a saber:

a. Episodios en los que el docente no considera las producciones matemáticas que los estudiantes generan en el desarrollo de tareas matemáticas.

b. Episodios en los que el docente considera las producciones matemáticas generadas por los estudiantes, sin embargo, posteriormente son invalidadas de forma arbitraria por el docente independientemente si estás producciones responden a la tarea matemática solicitada.

c. Finalmente, destaca la existencia de episodios en los cuales, además de la ejecución de la invalidación de las producciones generadas por los estudiantes, el docente impone arbitrariamente una forma de pensar y ejecutar la tarea presentada, la cual debe ser aceptada y replicada por los estudiantes sin necesidad de un análisis previo.

A continuación se presentará diferentes acciones que dan muestra del proceso de violencia simbólica ejercida en el proceso enseñanza aprendizaje en el contexto de educación matemática para 5to básico en establecimientos vulnerables.

Durante el proceso de análisis de uno de los videos recolectados, el docente solicita la verbalización de procedimientos matemáticos a ser utilizados en la resolución de una situación problemática, por parte de sus alumnos.

Dicha actividad no consideró las respuestas entregadas de los alumnos, lo cual genera que el profesor imponga procedimientos arbitrarios, los cuales en algún momento se contradicen con las instrucciones generadas por el docente. Lo anteriormente explicitado se ve reflejada en las siguientes expresiones del docente: 
“ ...y aquí nos vamos a ayudar con una recta numérica y ustedes van a ir viendo y después vamos a ir respondiendo, ya! Van a ir viendo la recta numérica, analizando la situación y después van responder ya! ¿Qué van a hacer primero entonces? ¡Van a leer! ¿Bryan que vas a hacer tú? ¡Vas a leer!.”

De igual forma se manifiesta la nula importancia que da la profesora a la producción estudiantil lo cual se traduce en la imposición de la respuesta requerida por el docente, en los siguientes diálogos que se plantean en el contexto de la revisión del trabajo realizado por grupos de estudiantes respecto del contenido "múltiplos de un número". Dicha corrección es guiada por la profesora la cual solicita a distintos estudiantes que socialicen sus respuestas usando el pizarrón.

\section{Diálogo 1:}

A partir de la siguiente exposición: “¿en qué fecha del mes se encuentran practicando juntos?"

Ahora vamos a empezar a contar el problema, necesito que un niñito de allá me va a responder algo...

A ver Iván, usted me dice en qué fecha del mes se encuentran practicando juntos, ¿lo quiere escribir? ¿Pensó bien primero lo que va a escribir?

¡Ya! escriba en qué fecha. Va a poner los días (afirmando) ¿cierto? La fecha del mes. (El alumno parte redactando la respuesta en pizarra).

Pero hijo, póngalo en numerito no más. (El alumno borra con su mano parte de las palabras escritas), (al ver la producción del alumno la profesora expresa)

“¿En el veinte no más se encuentran por primera vez? (la profesora toma el borrador a espaldas del niño y borra completamente la producción del estudiantes que incluía dos palabras distintas al 20).

(La profesora entrega una instrucción en voz baja al estudiante, logrando que éste escriba solo el 20 en la pizarra).

Luego afirma ¿parece que un niño encontró más? ¿A ver? (el alumno se queda parado al costado de la pizarra con el plumón en la mano, el cual es quitado por la profesora y el estudiante vuelve a su asiento).

En el presente diálogo se puede observar la profesora solicta la participación de los estudiantes intencionando una acción tendiente a considerar las producciones matemáticas que los estudiantes generan, sin embargo, posteriormente éstas son invalidadas de forma arbitraria por el docente incluso cuando la producción pesentada por el alumno respondía a la tarea matemática solicitada.

Finalmente es importante mencionar que la ejecución de la invalidación de las producciones generadas en este diálogo y la posterior imposición de la forma de presentar la respuesta en pizarra, no manifiesta explicitamente la imposición de una forma de pensar y ejecutar la tarea presentada por parte de la profesora. En este caso la imposición se centra en la forma en que se manifiesta el razonamiento del estudiantes, pero no explicitamente en la determinación de éste.

\section{Diálogo 2}

La profesora invita a una estudiante a responder a la tarea: "Escribe los múltiplos comunes de 5 y 10 ".

"A ver venga Constanza, a ver ¿pensaste bien Constanza?, ella va a escribir múltiplos comunes de 5 y 10 , (la alumna recibe el lápiz y la profesora le indica dónde debe escribir su respuesta), (la estudiante comienza a escribir su producción la cual consiste en un listado de múltiplos de 5 , mientras la profesora la acompaña detrás de ella. De improviso la profesora verbaliza en voz baja "comunes" y simultáneamente indica con el dedo la palabra "múltiplos comunes" de la pregunta, luego la profesora se da vuelta dando la espalda a la alumna que permanece frente a la pizarra, hace callar a los alumnos, toma el borrador y continua diciendo con voz fuerte ¡comunes!).

Ya, vaya a sentarse. (la alumna mira a la profesora y le entrega el plumón).

En este instante la profesora retoma su preguntas y la direcciona a otra alumna y borrando la producción realizada por la alumna en la pizarra.

Lo anterior refleja el criterio de la profesora para mostrar al estudiante que su producción es incorrecta, impidiendo que continúe escribiendo y mandándola a su puesto lo que impide que la estudiante presente su producción de forma completa al resto del curso. En consecuencia no se aprecia un argumento matemático que permita invalidar la respuesta de la estudiante, sino que lo realiza basado en su autoridad. En este 
contexto de identificación y caracterización de las manifestaciones de violencia simbólica producto de la acción docente, podemos describir la situación en la que:

La profesora empieza a revisar las producciones de los alumnos en la pizarra.

Cada alumno iba y escribía un múltiplo que se generaba a partir del trabajo de contabilizar grupos de legos.

En la pizarra está escrito:

$3=\{3,6,9,12,15,18,21,27,30,33,39,42,45,48,51,55,58,61,64,67 \ldots$.

El trabajo realizado presenta una extensión de la violencia simbólica pues se instala un procedimiento sin justificación, que no responde a una necesidad gestionada en la clase para analizar las respuestas de los alumnos, y establecer de esa forma si 66 ó 67 es verdaderamente múltiplo de 3. Lo anterior queda reflejado en la forma en que la profesora presenta el procedimiento respectivo a sus estudiantes:

"La profesora expone el siguiente procedimiento: $6+6=12$ y 12 está en la tabla del 3, por lo tanto 66 es múltiplo de 3. $6+7=13$ que no está en la tabla del 3 por lo tanto 67 no es múltiplo de 3. "

La profesora en consecuencia instaura un procedimiento, induciendo casi con carácter obligatorio sin ninguna justificación y necesidad; para que los alumnos lo utilicen hacen de este hecho una imposición arbitraria de un procedimiento matemático, ya que se trata de introducir el resultado de razonamiento matemático (que no visibiliza el profesor) sin valor de uso para el estudiante, sin contexto y por ende lo obliga a ejecutar pasos que no entiende pero, que producto de su autoridad, los sigue. Esta situación de imposición es de carácter simbólico pues ninguno de los alumnos reciente dicha imposición y genera en los estudiantes una validación de lo expuesto por la profesora sin reflexión observable, lo cual los lleva a manifestar en ejercicios posteriores de similar condición, cuando por ejemplo se requiere saber si 84 es múltiplo de 3 , un estudiante responde qué:

"Entonces como 84 es 12, y 12 está en la tabla del 3 entonces 84 es múltiplo de 3".

Con esto la profesora empieza a comprobar si 55 es múltiplo de 3 y así los alumnos comienzan a aplicar un procedimiento dado.

Ante un nuevo escenario la profesora pregunta sobre lo que hicieron la clase anterior, pero no utiliza las respuestas de los alumnos: aunque escucha a los alumnos, no valida, ni discute, ni refuta lo que ellos dicen.

De igual forma la profesora pregunta sobre el concepto de divisor, pero invalida las producciones de los alumnos:

"La profesora cambia la pregunta: "A ver, ¿qué son los divisores?" como una forma de acceder a la respuesta que ella espera.

Ante el silencio de los alumnos, dice “iPrimera clase!”, señalándoles dónde está la respuesta que ella espera. Es probable que este silencio se deba a que la profesora ignoró respuestas previas."

La profesora reitera la pregunta ¿Qué son los divisores?, algunos alumnos dan respuesta y la profesora las ignora.

Otro alumno levanta la voz al responder, para llamar su atención ("son los que dividen"); ante ello la profesora le dice: "ya, pero dígalo con sus propias palabras".

En consecuencia la gestión de la profesora busca imponer la respuesta: "un número es divisor de otro cuando la división es exacta", ignorando e invalidando las respuestas de los alumnos.De la misma manera la profesora pregunta qué es un múltiplo, pero no hace nada con las respuestas de los alumnos: aunque escucha a los alumnos, no valida, ni discute, ni refuta lo que ellos dicen.

La profesora abandona la pregunta "¿qué son los múltiplos?", ignorando con ello todas las producciones de los alumnos. En vez de ello, propone construir un ejemplo que ella inicia, imponiendo con ello el procedimiento.

Con lo anterior, cambia arbitrariamente el foco o propósito de esta acción, lo cual se presenta a continuación: 
"La profesora pide que los alumnos señalen cuáles son los múltiplos de 7. Ella dicta los dos primeros términos del conjunto: "Siete, catorce,...." Uno o dos niños alcanzan a decir esos números. (No se aprecia que la profesora considere las respuestas del curso o de estos niños).

Un niño señala que la secuencia de múltiplos continúa, diciendo "etcétera, etcétera".

La profesora dice "Ahhh,ya!!... ¿y cómo colocaba ese etcétera?”,

A lo que algunos niños responden "E-T-C", pero la profesora los ignora y vuelve a preguntar hasta que recibe la respuesta "con un punto, dos puntos, tres puntos".

Entonces ella afirma "con puntos sus-pen-sivos".

A continuación, la profesora dice "y los múltiplos dijimos que eran...".

Un alumno responde "muchos", pero la profesora vuelve a decir "...eran....".

Se produce un silencio la profesora dice "iiiin...", a lo que los alumnos responden "infinitos!!" y ella reafirma la respuesta.

Por lo tanto se observa una imposición por parte del docente señalando que eso se dice "infinito", y que se escribe con puntos suspensivos, ignorando las respuestas de los niños cuando señalan algo distinto a lo esperado.

\section{DISCUSIÓN}

En relación al análisis de los resultados asociados a los propósitos de la investigación y su conexión con los elementos teóricos se comentan las preguntas planteadas en la Introducción:

¿Qué caracteriza a las manifestaciones de violencia simbólica en las clases de matemática de 5to año básico en establecimientos de vulnerabilidad social?

1.- Una característica de la relación profesor-estudiante-actividad observada en clases de matemática está dada por un clima marcado por la neutralidad de las interacciones entre estudiantes y profesor en el desarrollo de cada actividad. En este sentido los estudiantes no manifiestan modificaciones notorias en su actitud pasiva cuando el docente les solicita la realización de alguna tarea matemática específica, dicha actitud se mantiene incluso en aquellos estudiantes que no hacen el esfuerzo por responder a la solicitud del docente. El profesor contribuye a esta relación de estabilidad aparente no relacionándose con los estudiantes que deciden no ejecutar la tarea, estableciéndose diálogos solamente con aquellos alumnos que intentar responder a la tarea planteada por el profesor y aquellos que sí interactúan lo hacen adaptándose a lo que el docente quiere que le respondan.

2.- La gestión de clases está centrada en "pasar la materia" y no en lo que los alumnos aprendan. Es así como podemos ver gestiones unidireccionales de clases en donde está claramente impuesta la creencia que "para aprender matemática el docente primero tiene que mostrar cómo se hace", además los errores se señalan (algunos) pero no se gestionan para el logro de los aprendizajes.

3.- En el proceso de caracterización de eventos de episodios de violencia simbólica en clase de matemática, se emergieron situaciones asociadas a eventos de violencia simbólica que enfatizan la no consideración de las producciones elaboradas por los estudiantes. Cabe destacar que solo en algunas situaciones de invalidación están además acompañadas por imposición arbitraria de conocimiento matemático. Algunas de estas variaciones se caracterizan por:

a. Episodios en los que el docente no considera las producciones matemáticas que los estudiantes generan en el desarrollo de tareas matemáticas.

b. Episodios en los que el docente considera las producciones matemáticas generadas por los estudiantes, sin embargo, posteriormente son invalidadas de

c. forma arbitraria por el docente independientemente si estás producciones responden correctamente a la tarea matemática solicitada.

d. Episodios en los cuales, además de la ejecución de la invalidación de las producciones generadas por los estudiantes, el docente impone arbitrariamente una forma de pensar y ejecutar la tarea presentada, la cual debe ser aceptada y replicada por los estudiantes sin necesidad de un análisis previo.

¿Existe una contradicción entre el habitus del alumno respecto de su razonamiento matemático y la forma como el profesor enseña la matemática del currículum escolar? 
1.- Según lo observado en los videos, los docentes de escuelas vulnerables no comprenden que los contenidos matemáticos son un medio para el logro de aprendizajes curriculares que permitan a los alumnos abordar y resolver problemas con valor de uso del conocimiento. Los contextos que se ofrecen son intramatemáticos, o bien, son contextos muy cercanos al alumno pero relacionados de forma espuria con el contenido matemático. Además, el tratamiento de los contenidos se reduce sólo a la verificación de las relaciones matemáticas.

2.- Desde esta perspectiva en las clases de matemática se observa contradicción entre el habitus del estudiante y la forma en que el profesor enseña matemática. Dicha contradicción se manifiesta en lo que el profesor espera que su estudiante manifieste como aprendizaje matemático y lo que el estudiante efectivamente manifiesta en la sala de clases. Específicamente el profesor no reconoce en las producciones elaboradas por el estudiante, formas de razonamiento que pueden ser utilizadas para construir conocimiento matemático en las clases. En dicha situaciones los aportes que emergen de los estudiantes en las clases de matemática acrecen de valor para el profesor por tanto se invalida la forma en que el estudiante estructura el conocimiento matemático.

3.- Desde el punto de vista de análisis lingüístico propuesto por Bourdieu se observa que el habitus lingüístico del estudiante es muchas veces invisibilizado en la clase de matemática, y en casos como el verificado en el diálogo 1, difícil de apreciar ya que en las clases observada no existe la posibilidad de establecer incluso un diálogo. En otras situaciones, el habitus lingüístico del estudiante en el mercado lingüístico determinado por el profesor en la sala de clases, manifiesta una falta de valoración de la argumentación entregada por el estudiante en el contexto de interacción profesor alumno. Estas manifestaciones están acompañadas por relaciones de dominación ejercida por el profesor(a) en el proceso de enseñanza de la matemática, las cuales que se traducen en presentar instrucciones a los estudiantes que declaran implícitamente la forma en que la opinión debe ser entregada al profesor o al curso para que ésta sea valorada en la clases. Estas manifestaciones y contradicciones posiblemente se presenten en diversos ámbitos escolares, incluso en el contexto universitario.

¿Cómo se manifiesta la contradicción entre el habitus del alumno respecto a su razonamiento matemático y la actividad didáctica realizada por el profesor en la implementación del currículum escolar?

1.- No se generan condiciones para que los alumnos propongan y ensayen procedimientos que posteriormente sean modificados por otros más eficaces y que sean los propios alumnos quienes expliciten en forma verbal y escrita las explicaciones de por qué algunos procedimientos son poco eficientes. Así mismo no se aprecia la generación de una discusión basada en la interacción lingüística fundamentalmente verbal, que posibilite permear el plano didáctico con argumentos presentados por los estudiantes desde su propio habitus. Esto trae consigo un deterioro en la apropiación del conocimiento matemático y una distorsión de lo que significa aprender matemática.

2.-Así la manifestación de la relación dialéctica entre la cultura escolar y el capital cultural con que llegan a la escuela el estudiante, tiende a invisibilizarse producto de la gestión inadecuada de las variables didácticas en el contexto del aprendizaje matemático.

3.- Respecto a lo anterior en relación a la invalidación de los resultados de las tareas matemáticas observadas en clases y asumiendo que según Chevallard (1999) toda producción realizada en un contexto de escolares referida a una tarea matemática requiere de la articulación de una razonamiento matemáticos para ser elaborada, se considera que el profesor cuando invalida o no considera las respuestas de los estudiantes en la creación conjunta de conocimientos, lo que se está invalidando finalmente es La manifestación del razonamiento matemático del estudiantes.

Del análisis anterior es posible encontrar elementos que perfectamente podrían estar presentes en otros contextos y niveles de formación, por ejemplo en el caso de los tipos y niveles de interacción que se observan entre alumnos y docentes a nivel universitario, en donde es posible apreciar gestiones unidireccionales de clases poniendo de manifiesto la imposición en el proceso de formación universitaria. Lo anteriormente planteado se verifica principalmente por "la creencia mayoritaria, en la cultura iberoamericana, de que una sólida formación científica y profesional le basta al docente para transmitir los contenidos de su asignatura, desestimándose los saberes pedagógicos" (Krzemien \& Lombardo, 2006, p. 1).

Sería importante estudiar con mayor profundidad las caracterizaciones y contradicciones encontradas en esta investigación que permitan justificar por qué incluso en la formación universitaria podría ser posible el mejoramiento de los aprendizajes así como los altos índices de deserción en los primeros años de formación a partir de la "generación de un contexto comunicativo acogedor y amistoso, basado en el respeto 
mutuo entre todos y la abstinencia de acciones que intimiden a los Alumnos" (Al-Hamdan, 2007). Es sabido que se aprende más y mejor cuando en el aula se asiste con agrado, cuando se percibe buen trato desde sus educadores, cuando cada uno se siente valorado y reconocido (Bourgeois \& Nizet, 1997, en Sebastián, 2007), sin embargo el reconocimiento debe ser comprendido en una expresión amplia que incluya las distintas formas de razonamiento de los estudiantes.

\section{CONCLUSIONES}

Con base en los resultados y la discusión de la sección anterior se puede concluir lo siguiente:

En relación al análisis de los resultados asociados a los propósitos de la investigación y su conexión con los elementos teóricos es posible concluir que:

1.- Una característica de la relación profesor-estudiante-actividad observada en clases de matemática está dada por un clima marcado por la neutralidad de las interacciones entre estudiantes y profesor en el desarrollo de cada actividad.

2.- La gestión de clases está centrada en "pasar la materia" y no en lo que los alumnos aprendan.

3.- En el proceso de caracterización de eventos de episodios de violencia simbólica en clase de matemática, se emergieron situaciones asociadas a eventos de violencia simbólica que enfatizan la no consideración de las producciones elaboradas por los estudiantes.

4.- Los docentes de escuelas vulnerables no comprenden que los contenidos matemáticos son un medio para el logro de aprendizajes curriculares que permitan a los alumnos abordar y resolver problemas con valor de uso del conocimiento.

5.- Desde esta perspectiva en las clases de matemática se observa contradicción entre el habitus del estudiante y la forma en que el profesor enseña matemática.

6.- No se generan condiciones para que los alumnos propongan y ensayen procedimientos que posteriormente sean modificados por otros más eficaces y que sean los propios alumnos quienes expliciten en forma verbal y escrita las explicaciones de por qué algunos procedimientos son poco eficientes.

\section{AGRADECIMIENTOS}

Este artículo no habría sido posible sin la valiosa colaboración de los profesores Rodrigo Ulloa Sánchez y Milton Sepúlveda Ahumada quienes aportaron en el trabajo de campo y en la discusión socializada de este artículo.

\section{REFERENCIAS}

Alonso, L. "Pierre Bourdieu, El lenguaje y la comunicación: De los mercados lingüísticos a la degradación Mediática.", en Moreno, L. "Pierre Bourdieu : las herramientas del sociólogo". España, editorial Fundamentos. Pp. 215-254.(2004).

Al-Hamdan, J. "Higher Education Classroom Management: Kuwait University Student's Views", en college student Journal, Vol. 41, (3), pp. 527-582. (2007).

Ausubel, D.. "Adquisición y retención del conocimiento. Una perspectiva cognitiva". Barcelona: Paidós. (2002).

Bourdieu, J.."La Reproducción”. Barcelona: España, Editorial Laia. (1977).

Bourdieu, P. “¿Qué significa Hablar?. Economía de los Intercambio lingüísticos”, Madrid, Akal. (1985)

Bourdieu J, y Passeron J.. " La Reproducción; elementos para una teoría del sistema de enseñanza". Ediciones Fontamara México. (2005).

Castro. A."Proyecto Anillos de investigación SOC 15: "Dialéctica de los Aprendizajes y ruptura del círculo de bajos aprendizajes en sectores de alta vulnerabilidad." Programa CONICYT Bicentenario de Ciencia y tecnología. Concepción. (2009). 
Chevallard, Y. "El análisis de las prácticas docentes en la teoría antropológica de lo didáctico". Recherches en Didactique des Mathématiques, Vol 19, nº 2, pp. 221-266, (1999).

Krzemien, Deisy \& Lombardo, E. "Rol Docente universitario y Competencias Profesionales en la Licenciatura de Psicología”, en Psicología Escolar e Educacional. Vol. 10, (2), pp. 173-186. (2006).

MINEDUC Ministerio de Educación “Informe SIMCE Unidad de Curriculum y Evaluación”. Santiago de Chile. (2008).

MINEDUC Ministerio de Educación “Informe SIMCE Unidad de Curriculum y Evaluación. Santiago de Chile. (2010).

Sebastián, C. "La diversidad interindividual como una oportunidad para el aprendizaje de los estudiantes de educación superior". en revista calidad en la Educación, (26), pp. 8-101. (2007).

Villalta, M.; Martinic, S. "Modelos de estudio de la interacción didáctica en la sala de clase". Investigación y Postgrado, Caracas, v. 24, n. 2. (2009).

Willis, P. "Aprendiendo a trabajar: Cómo los chicos de la clase obrera consiguen trabajo de la clase obrera". Madrid, Akal. (1988). 